\title{
Análise de convergência de métodos proximais para soluções eficientes em otimização multiobjetivo convexa
}

\author{
Rogério Azevedo Rocha ${ }^{1}$ \\ Curso de Ciênca da Computação, UFT, Palmas, TO \\ Ronaldo Malheiros Gregório ${ }^{2}$ \\ Departamento de Tecnologia e Linguagens, UFRRJ, Rio de Janeiro, RJ \\ Michael Souza ${ }^{3}$ \\ Departamento de Estatística e Matemática Aplicada, UFC, Ceará, CE
}

Resumo. Recentemente Rocha et al. construíram um método de escalarização proximal para problemas de otimização multiobjetivo irrestrito e convexo, onde os autores provam a convergência do método para soluções fracamente eficientes. Dado que em aplicações reais é frequente o caso em que apenas soluções eficientes (em vez de fracamente eficientes) são de interesse, neste trabalho investigamos a convergência do método de Rocha et al. para soluções eficientes.

Palavras-chave. Otimização Multiobjetivo, Algoritmo Proximal, Quase-distância, Solução eficiente

\section{Introdução}

Este trabalho considera o problema de otimização multiobjetivo (POM) irrestrito

$$
\text { MINIMIZE }\left\{F(x) \mid x \in \mathbb{R}^{n}\right\}
$$

onde $F=\left(F_{1}, F_{2}, \ldots, F_{m}\right)^{T}: \mathbb{R}^{n} \rightarrow \mathbb{R}^{m}$ satisfaz as seguintes hipóteses: (H) $\left.\mathbf{1}\right) F$ é convexa e ( $\mathcal{H} 2) F$ possui pelo menos uma de suas funções objetivo coerciva, i.e., existe $r \in\{1, \ldots, m\}$ tal que $\lim F_{r}(x)=+\infty$ quando $\|x\| \rightarrow+\infty$.

A importância da otimização multiobjetivo pode ser conferida em uma grande variedade de aplicações presentes na literatura. White [8] oferece uma bibliografia de 504 artigos descrevendo várias aplicações que abordam, por exemplo, problemas relacionados à agricultura, serviços bancários, serviços de saúde e energia. Mais informações, com respeito a otimização multiobjetivo, podem ser conferidas na Seção 2 e Mitettinen [3].

Gregório e Oliveira [1] apresentaram um método de escalarização ponto proximal para o problema (1). Neste trabalho os autores provam a convergência do método para soluções fracamente eficientes. Este método de Gregório e Oliveira foi generalizado por Rocha et

\footnotetext{
11azevedo@uft.edu.br

${ }^{2}$ rgregor@ufrrj.br

${ }^{3}$ michael@ufc.br
} 
al. [6] onde foi considerado uma quase-distância em substituição ao termo quadrático do método de Gregório e Oliveira. Assim como Gregório e Oliveira, Rocha et al. provam a convergência de seu método para soluções fracamente eficientes.

Neste trabalho, mostraremos que o método proximal de Rocha et al. [6] converge para soluções eficientes. Neste sentido, concluímos também que o mesmo ocorre com o algoritmo de Gregório e Oliveira [1].

A principal justificativa da importância deste trabalho é que, em aplicações reais é frequente o caso em que apenas soluções eficientes são de interesse (ver, por exemplo, Seção 2.3 em [2]). Portanto, como para os métodos de Gregório e Oliveira [1] e Rocha et al. [6] é garantido somente convergência para soluções fracamente eficientes, nosso trabalho estende estes trabalhos anteriores em relação ao conceito de solução.

\section{Preliminares}

Nesta Seção recordaremos algumas propriedades dos subdiferenciais Fréchet e limite e da aplicação quase-distância. Além disto, faremos uma breve revisão da programação multiobjetivo. Mais detalhes podem ser conferidos em [3], [4] e [7].

Definição 2.1. Sejam $x \in \mathbb{R}^{n}$ e $h: \mathbb{R}^{n} \rightarrow \mathbb{R} \cup\{+\infty\}$ uma função própria e semicontínua inferior.

a) O subdiferencial Fréchet de $h$ em $x$, $̂ h(x)$, é dado por:

$$
\hat{\partial} h(x):= \begin{cases}\left\{x^{*} \in \mathbb{R}^{n} \mid \liminf _{y \neq x, y \rightarrow x} \frac{h(y)-h(x)-\left\langle x^{*}, y-x\right\rangle}{\|x-y\|} \geq 0\right\}, & \text { se } x \in \operatorname{dom}(h) \\ \varnothing, & \text { se } x \notin \operatorname{dom}(h)\end{cases}
$$

b) O subdiferencial-limite de $h$ em $x, \partial h(x)$, é dado por:

$$
\partial h(x):=\left\{x^{*} \in \mathbb{R}^{n} \mid \exists x_{n} \rightarrow x, \quad h\left(x_{n}\right) \rightarrow h(x), \quad x_{n}^{*} \in \hat{\partial} h\left(x_{n}\right) \text { e } x_{n}^{*} \rightarrow x^{*}\right\}
$$

Proposição 2.1. Para uma função $h: \mathbb{R}^{n} \rightarrow \mathbb{R} \cup\{+\infty\}$ e um ponto $\bar{x} \in \operatorname{dom}(h)$, os conjuntos $\hat{\partial} h(\bar{x})$ e $\partial h(\bar{x})$ são fechados, com $\hat{\partial} h(\bar{x})$ convexo e $\hat{\partial} h(\bar{x}) \subset \partial h(\bar{x})$.

Demonstração. Rockafellar e Wets [7], Teorema 8.6.

Proposição 2.2. Se uma função própria $h: \mathbb{R}^{n} \rightarrow \mathbb{R} \cup\{+\infty\}$ possui um mínimo local em $\bar{x} \in \operatorname{dom}(h)$, então $0 \in \hat{\partial} h(\bar{x})$ e $0 \in \partial h(\bar{x})$.

Demonstração. Rockafellar e Wets [7], Teorema 10.1.

Observação 2.1. Seja $C \subset \mathbb{R}^{n}$. Se uma função própria $h: C \rightarrow \mathbb{R} \cup\{+\infty\}$ possui um mínimo local $\bar{x} \in C$, então $0 \in \hat{\partial}\left(h+\delta_{C}\right)(\bar{x}), 0 \in \partial\left(h+\delta_{C}\right)(\bar{x})$, onde $\delta_{C}$ é a função indicatora do conjunto $C$.

Em seguida vamos definir a aplicação quase-distância que será utilizada como parte da regularização dos subproblemas do algoritmo que iremos propor na próxima Seção. 
Definição 2.2. Uma aplicação $q: \mathbb{R}^{n} \times \mathbb{R}^{n} \rightarrow \mathbb{R}_{+}$é uma quase-distância em $\mathbb{R}^{n}$ se, para todos $x, y, z \in \mathbb{R}^{n}$,
a) $q(x, y)=q(y, x)=0 \Longleftrightarrow x=y$
b) $q(x, z) \leq q(x, y)+q(y, z)$.

Proposição 2.3. Seja $q: \mathbb{R}^{n} \times \mathbb{R}^{n} \rightarrow \mathbb{R}_{+}$uma quase-distância em $\mathbb{R}^{n}$. Suponha que existem constantes positivas $\alpha$ e $\beta$ tais que

$$
\alpha\|x-y\| \leq q(x, y) \leq \beta\|x-y\|, \quad \forall x, y \in \mathbb{R}^{n} .
$$

Então, para cada $\bar{z} \in \mathbb{R}^{n}$, a) As funções $q(\bar{z},$.$) e q(., \bar{z})$ são lipschitzianas em $\mathbb{R}^{n}$, b) As funções $q^{2}(\bar{z},$.$) e q^{2}(., \bar{z})$ são localmente lipschitzianas em $\mathbb{R}^{n}$ e c) As funções $q(\bar{z},$.$) ,$ $q(., \bar{z}), q^{2}(\bar{z},$.$) e q^{2}(., \bar{z})$ são coercivas.

Demonstração. Moreno et al. [5], Proposições 3.6 e 3.7 e Observação 5.

Antes de definirmos os conceitos de solução para problemas multiobjetivo apresentamos as seguintes notações.

Notação 2.1. Considere os vetores $y, \bar{y} \in \mathbb{R}^{m}$. Utilizaremos as seguintes notações: a) $\left.y \leq \bar{y} \Longleftrightarrow y_{i} \leq \bar{y}_{i} \forall i=1, \ldots, m ; \boldsymbol{b}\right) y<\bar{y} \Longleftrightarrow y_{i} \leq \bar{y}_{i} \forall i=1, \ldots, m$, com a desigualdade estrita assegurada para pelo menos um indice e c) $y \ll \bar{y} \Longleftrightarrow y_{i}<\bar{y}_{i} \quad \forall i=1, \ldots, m$.

Considere uma aplicação multiobjetivo $G: \mathbb{R}^{n} \longrightarrow \mathbb{R}^{m}$ e o seguinte Problema de Otimização Multiobjetivo (POM) irrestrito

$$
\operatorname{MiNIMIZE}\left\{G(x) \mid x \in \mathbb{R}^{n}\right\} \text {. }
$$

Definição 2.3. a) Dizemos que $a \in \mathbb{R}^{n}$ é uma solução eficiente local para o problema (3) se existe um disco $B_{\delta}(a) \subset \mathbb{R}^{n}$, com $\delta>0$, tal que não existe $x \in B_{\delta}(a)$ satisfazendo $G(x)<G(a)$ e, b) Dizemos que $a \in \mathbb{R}^{n}$ é uma solução fracamente eficiente local para o problema (3) se existe um disco $B_{\delta}(a) \subset \mathbb{R}^{n}$, com $\delta>0$, tal que não existe $x \in B_{\delta}(a)$ satisfazendo $G(x) \ll G(a)$.

Denotemos por ARG $\operatorname{MIN}\left\{G(x) \mid x \in \mathbb{R}^{n}\right\}$ e ARG $\operatorname{MIN}_{w}\left\{G(x) \mid x \in \mathbb{R}^{n}\right\}$ os conjuntos das soluções eficientes locais e soluções fracamente eficientes locais para o problema (3), respectivamente. É fácil ver que ARG $\operatorname{MIN}\left\{G(x) \mid x \in \mathbb{R}^{n}\right\} \subset \operatorname{ARG} \operatorname{MIN}_{w}\left\{G(x) \mid x \in \mathbb{R}^{n}\right\}$.

Em geral, se um problema de otimização multiobjetivo restrito ou irrestrito é um problema convexo, i.e., se a função objetivo $G: \mathbb{R}^{n} \rightarrow \mathbb{R}^{m}$ é uma função convexa, então toda solução (fracamente) eficiente local é também uma solução (fracamente) eficiente global. Este resultado é discutido no Teorema 2.2.3, em Miettinen [3].

Definição 2.4. Uma função de valor escalar $g: \mathbb{R}^{n} \longrightarrow \mathbb{R}$ é dita ser uma

a) Representação escalar de uma aplicação $G: \mathbb{R}^{n} \longrightarrow \mathbb{R}^{m}$ quando, dados $x, \bar{x} \in \mathbb{R}^{n}$, $G(x) \leq G(\bar{x}) \Longrightarrow g(x) \leq g(\bar{x}) \quad$ e $G(x)<G(\bar{x}) \Longrightarrow g(x)<g(\bar{x}) ;$

b) Representação escalar estrita de uma aplicação $G: \mathbb{R}^{n} \longrightarrow \mathbb{R}^{m}$ quando, dados $x, \bar{x} \in \mathbb{R}^{n}, \quad G(x) \leq G(\bar{x}) \Longrightarrow g(x) \leq g(\bar{x}) \quad$ e $G(x) \ll G(\bar{x}) \Longrightarrow g(x)<g(\bar{x}) ;$ 
c) Representação escalar fraca de uma aplicação $G: \mathbb{R}^{n} \longrightarrow \mathbb{R}^{m}$ quando, dados $x, \bar{x} \in \mathbb{R}^{n}, \quad G(x) \ll G(\bar{x}) \Longrightarrow g(x)<g(\bar{x})$.

Segue imediatamente da definição 2.4 acima: a) $\Rightarrow$ b) $\Rightarrow$ c).

Dados $g: \mathbb{R}^{n} \rightarrow \mathbb{R}$ e $\Omega \subseteq \mathbb{R}^{n}$ considere arg $\min \{g(x) \mid x \in \Omega\}$ denotanto o conjunto dos minimizadores locais de $g$ em $\Omega$. Segue da Definição 2.4 que

$$
\arg \min \{g(x) \mid x \in \Omega\} \subset \mathrm{ARG} \operatorname{MIN}_{w}\{G(x) \mid x \in \Omega\} .
$$

\section{Convergência para soluções eficientes}

Rocha et al [6] supuseram a existência de uma aplicação $f: \mathbb{R}^{n} \times \mathbb{R}_{+}^{m} \longrightarrow \mathbb{R}$ satisfazendo as propriedades $(P 1)$ à $(P 4)$ abaixo e então provaram a convergência do método de escalarização proximal para soluções fracamente eficientes do POM (1). Seguem as propriedades.

(P1) $f$ é limitada inferiormente por algum $\alpha \in \mathbb{R}$;

(P2) $f$ é convexa em $\mathbb{R}^{n} \times \mathbb{R}_{+}^{m}$;

(P3) $f$ é uma representação escalar estrita de $F$, com respeito a $x$, i.e.,

$$
F(x) \leq F(y) \Rightarrow f(x, z) \leq f(y, z) \quad \text { e } F(x) \ll F(y) \Rightarrow f(x, z)<f(y, z)
$$

para todos $x, y \in \mathbb{R}^{n}$ e $z \in \mathbb{R}_{+}^{m}$;

(P4) $f$ é diferenciável, com respeito a $z$ e

$$
\frac{\partial}{\partial z} f(x, z)=h(x, z)
$$

onde $h(x, z)=\left(h_{1}(x, z), \cdots, h_{m}(x, z)\right)^{T}$ é uma aplicação contínua de $\mathbb{R}^{n} \times \mathbb{R}^{m}$ para $\mathbb{R}_{+}^{m}$, i.e, $h_{i}(x, z) \geq 0$ para todo $i=1, \cdots, m$.

Mostraremos nesta seção que se a propriedade (P3) for substituída pela propriedade

(P3)' $f$ é uma representação escalar de $F$, com respeito a $x$, i.e., $\forall x, y \in \mathbb{R}^{n}$ e $z \in \mathbb{R}_{+}^{m}$,

$$
F(x) \leq F(y) \Rightarrow f(x, z) \leq f(y, z) \text { e } F(x)<F(y) \Rightarrow f(x, z)<f(y, z)
$$

então a convergência do método de Rocha et al. [6] é na verdade para soluções eficientes (em vez de soluções fracamente eficientes) do POM (1). Salientamos que os exemplos de aplicações apresentados por Gregório e Oliveira [1] e por Rocha et al. [6] que satisfazem as propriedades $(\mathrm{P} 1)$ à $(\mathrm{P} 4)$ também satisfazem a propriedade $(\mathrm{P} 3)^{\prime}$.

Seja $F: \mathbb{R}^{n} \rightarrow \mathbb{R}^{m}$ uma aplicação satisfazendo as hipóteses $(\mathcal{H} 1)$ e $(\mathcal{H} 2)$. Para a definição do método de escalarização proximal (método EPLQD), Rocha et al. [6] consideram as seguintes hipóteses. 
(a) $f: \mathbb{R}^{n} \times \mathbb{R}_{+}^{m} \longrightarrow \mathbb{R}$ satisfaz as propriedades (P1) à (P4);

(b) $q: \mathbb{R}^{n} \times \mathbb{R}^{n} \rightarrow \mathbb{R}_{+}$quase-distância atende (2);

(c) para $\bar{z} \in \mathbb{R}_{++}^{m}$ (fixo), $H_{\bar{z}}: \mathbb{R}_{++}^{m} \rightarrow \mathbb{R}$ é tal que $H_{\bar{z}}(z)=\left\langle\frac{z}{\bar{z}}-\log \frac{z}{\bar{z}}-e, e\right\rangle$, onde $e=(1, \ldots, 1) \in \mathbb{R}^{m}, \frac{z}{\bar{z}}=\left(\frac{z_{1}}{\bar{z}_{1}}, \ldots, \frac{z_{m}}{\bar{z}_{m}}\right)$ e $\log \frac{z}{\bar{z}}=\left(\log \frac{z_{1}}{\bar{z}_{1}}, \ldots, \log \frac{z_{m}}{\bar{z}_{m}}\right) ;$

(d) $\left\{\beta^{k}\right\}$ e $\left\{\mu^{k}\right\}$ são sequências de parâmetros reais que satisfazem: $\beta^{k}>0, \forall k \in \mathbb{N}$ e $0<l<\mu^{k}<L<+\infty, \forall k \in \mathbb{N}$.

O método de Rocha et al. [6] gera uma sequência $\left\{\left(x^{k}, z^{k}\right)\right\} \subset \mathbb{R}^{n} \times \mathbb{R}_{++}^{m}$ da seguinte forma:

\section{Método EPLQD}

1. Tome $x^{0} \in \mathbb{R}^{n}$ e $z^{0} \in \mathbb{R}_{++}^{m}$.

2. Dados $x^{k} \in \mathbb{R}^{n}$ e $z^{k} \in \mathbb{R}_{++}^{m}$, encontre $x^{k+1} \in \mathbb{R}^{n}$ e $z^{k+1} \in \mathbb{R}_{++}^{m}$ tais que

$$
\left(x^{k+1}, z^{k+1}\right) \in \arg \min \left\{\varphi^{k}(x, z) \mid(x, z) \in \Omega^{k} \times \mathbb{R}_{++}^{m}\right\},
$$

onde $\varphi^{k}(x, z)=f(x, z)+\beta^{k} H_{z^{k}}(z)+\frac{\mu^{k}}{2} q^{2}\left(x, x^{k}\right)$ e $\Omega^{k}=\left\{x \in \mathbb{R}^{n} \mid F(x) \leq F\left(x^{k}\right)\right\}$.

3. Se $\left(x^{k+1}, z^{k+1}\right)=\left(x^{k}, z^{k}\right)$, então pare ( pois $\left.x^{k} \in \operatorname{ARG} \operatorname{MIN}\left\{F(x) \mid x \in \mathbb{R}^{n}\right\}\right)$.

A partir de agora vamos considerar o método EPLQD com a aplicação $f: \mathbb{R}^{n} \times \mathbb{R}_{+}^{m} \longrightarrow$ $\mathbb{R}$ satisfazendo a propriedade (P3)' em substituição a propriedade (P3) e consequentemente provar a convergência do método EPLQD para soluções eficientes. É importante observar que tudo o que foi provado em Rocha et al. [6] continua válido pois a propriedade (P3)' implica na propriedade (P3). Sendo assim, nos limitaremos apenas a provar o resultado principal de convergência. Citaremos os outros resultados.

Lema 3.1. Seja $F=\left(F_{1}, F_{2}, \ldots, F_{m}\right)^{T}: \mathbb{R}^{n} \rightarrow \mathbb{R}^{m}$ uma aplicação satisfazendo as hipóteses $(\mathcal{H} 1)$ e (H2). Então, para cada $\bar{x} \in \mathbb{R}^{n}$ (fixado), o conjunto $\bar{\Omega}=\left\{x \in \mathbb{R}^{n} \mid F(x) \leq F(\bar{x})\right\}$ é convexo e compacto. Particularmente, $\bar{\Omega} \times \mathbb{R}_{+}^{m}$ é um conjunto convexo e fechado.

Demonstração. Rocha et al. [6], Lema 4.1.

Observação 3.1. Suponha que $\left\{x^{k}\right\}_{k \in \mathbb{N}}$ seja uma sequência gerada pelo método EPLQD. Pelo Lema 3.1, $\Omega^{k}, \forall k \in \mathbb{N}$ é um conjunto compacto. Portanto, como $\Omega^{k+1} \subseteq \Omega^{k}, \forall k \in \mathbb{N}$, temos: $\Omega=\bigcap_{k=0}^{\infty} \Omega^{k} \neq \emptyset$.

Proposição 3.1 (Boa Definição). Sejam $F=\left(F_{1}, F_{2}, \ldots, F_{m}\right)^{T}: \mathbb{R}^{n} \rightarrow \mathbb{R}^{m}$ uma aplicação

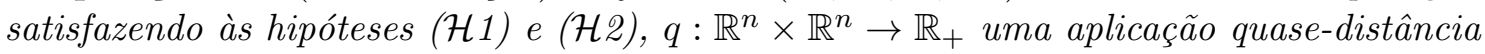
satisfazendo (2) e $f: \mathbb{R}^{n} \times \mathbb{R}_{+}^{m} \longrightarrow \mathbb{R}$ uma aplicação verificando as propriedades (P1), (P2), (P3)' e (P4). Então, para todo $k \in \mathbb{N}$, existe uma solução $\left(x^{k+1}, z^{k+1}\right)$ para $o$ problema (4). 
Demonstração. Rocha et al. [6], Proposição 4.2.

Proposição 3.2 (Critério de Parada). Seja $\left\{\left(x^{k}, z^{k}\right)\right\}_{k \in N}$ uma sequência gerada pelo método EPLQD. Se $\left(x^{k+1}, z^{k+1}\right)=\left(x^{k}, z^{k}\right)$ para algum inteiro $k$ então $x^{k}$ é uma solução eficiente (ou Pareto) para o POM irrestrito (1).

Demonstração. Análoga a prova da Proposição 4.3 de Rocha et al. [6].

Proposição 3.3 (Propriedades). Seja $\left\{\left(x^{k}, z^{k}\right)\right\}_{k \in \mathbb{N}}$ uma sequência gerada pelo método EPLQD. Então: i) $\left\{x^{k}\right\}_{k \in \mathbb{N}}$ é limitada; ii) $\left\{z^{k}\right\}_{k \in \mathbb{N}}$ é convergente; iii) $\left\{f\left(x^{k}, z^{k}\right)\right\}_{k \in \mathbb{N}}$ é não crescente e convergente; iv) $\sum_{k=0}^{+\infty} q^{2}\left(x^{k+1}, x^{k}\right)<+\infty$ e $\left.\boldsymbol{v}\right) \lim _{k \rightarrow+\infty}\left\|x^{k}-x^{k+1}\right\|=0$.

Demonstração. Rocha et al. [6], Proposições 4.4 e 4.5 .

Agora podemos provar a convergência do método EPLQD para soluções eficientes se o critério de parada nunca se aplica.

Teorema 3.1 (convergência). Sejam $F=\left(F_{1}, F_{2}, \ldots, F_{m}\right)^{T}: \mathbb{R}^{n} \rightarrow \mathbb{R}^{m}$ uma aplicação

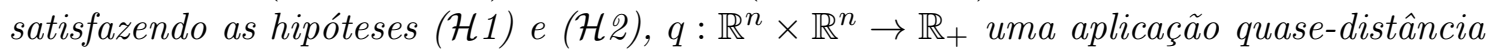
satisfazendo (2) e $f: \mathbb{R}^{n} \times \mathbb{R}_{+}^{m} \longrightarrow \mathbb{R}$ uma aplicação verificando as propriedades (P1), (P2), (P3)' e (P4). Então, qualquer sequência $\left\{\left(x^{k}, z^{k}\right)\right\}_{k \in \mathbb{N}} \subset \mathbb{R}^{n} \times \mathbb{R}_{++}^{m}$ gerada pelo método EPLQD satisfaz: $\left\{z^{k}\right\}_{k \in \mathbb{N}}$ é convergente e $\left\{x^{k}\right\}_{k \in \mathbb{N}}$ é limitada com seus pontos de acumulação sendo soluções eficientes (ou Pareto) para o POM (1).

Demonstração. Pela Proposição 3.3, $\left\{z^{k}\right\}_{k \in \mathbb{N}}$ é convergente e $\left\{x^{k}\right\}_{k \in \mathbb{N}}$ é limitada. Portanto, existem $x^{*} \in \mathbb{R}^{n}, z^{*} \in \mathbb{R}_{+}^{m}$ e $\left\{x^{k_{j}}\right\}_{j \in \mathbb{N}}$ subsequência de $\left\{x^{k}\right\}_{k \in \mathbb{N}}$ tal que $\lim _{j \rightarrow+\infty} x^{k_{j}}=$ $x^{*}$ e $\lim _{k \rightarrow+\infty} z^{k}=z^{*}$. Pela Proposição 3.3, $\left\{f\left(x^{k}, z^{k}\right)\right\}_{k \in \mathbb{N}}$ é não crescente e convergente. Por (P2), $f$ é contínua em $\mathbb{R}^{n} \times \mathbb{R}_{+}^{m}$. Logo,

$$
\lim _{k \rightarrow+\infty} f\left(x^{k}, z^{k}\right)=\lim _{j \rightarrow+\infty} f\left(x^{k_{j}}, z^{k_{j}}\right)=f\left(x^{*}, z^{*}\right)=\inf _{k \in \mathbb{N}}\left\{f\left(x^{k}, z^{k}\right)\right\} .
$$

Considere $N_{C}(\bar{x})=\left\{v \in \mathbb{R}^{n} \mid\langle v, x-\bar{x}\rangle \leq 0 \forall x \in C\right\}$ o cone normal no ponto $\bar{x}$ em relação ao conjunto convexo $C$ que contém $\bar{x}$. Pelo Corolário 4.1(i) de Rocha et al. [6], existem $\zeta^{k+1} \in \partial\left(q\left(., x^{k}\right)\right)\left(x^{k+1}\right)$ e $v^{k+1} \in N_{\Omega^{k}}\left(x^{k+1}\right)$ tais que

$$
-\mu^{k} q\left(x^{k+1}, x^{k}\right) \zeta^{k+1}-v^{k+1} \in \partial f\left(., z^{k+1}\right)\left(x^{k+1}\right) .
$$

Daí, pela desigualdade do subgradiente para a função convexa $f\left(., z^{k+1}\right)$ temos: $\forall x \in \mathbb{R}^{n}$,

$$
\begin{aligned}
f\left(x, z^{k_{j}+1}\right) & \geq f\left(x^{k_{j}+1}, z^{k_{j}+1}\right)-\mu^{k_{j}} q\left(x^{k_{j}+1}, x^{k_{j}}\right)\left\langle\zeta^{k_{j}+1}, x-x^{k_{j}+1}\right\rangle \\
& -\left\langle v^{k_{j}+1}, x-x^{k_{j}+1}\right\rangle
\end{aligned}
$$

Como $v^{k_{j}+1} \in N_{\Omega^{k_{j}}}\left(x^{k_{j}+1}\right)$ temos $-\left\langle v^{k_{j}+1}, x-x^{k_{j}+1}\right\rangle \geq 0, \quad \forall x \in \Omega^{k_{j}}$. Pela Observação 3.1, $\Omega=\bigcap_{k=0}^{\infty} \Omega^{k} \neq \emptyset$. Portanto, em particular, de (6) temos: $\forall x \in \Omega$,

$$
f\left(x, z^{k_{j}+1}\right) \geq f\left(x^{k_{j}+1}, z^{k_{j}+1}\right)-\mu^{k_{j}} q\left(x^{k_{j}+1}, x^{k_{j}}\right)\left\langle\zeta^{k_{j}+1}, x-x^{k_{j}+1}\right\rangle
$$


Pela Prop. 4.6 de Rocha et al. [6] e Prop. 3.3 (iv), $\left\|\zeta^{k_{j}+1}\right\| \leq M$ e $\lim _{k \rightarrow+\infty} q\left(x^{k+1}, x^{k}\right)=0$, respectivamente. Logo, como $0<l<\mu^{k}<L, \forall k \in \mathbb{N}$ e $\left\{x^{k}\right\}_{k \in \mathbb{N}}$ é limitada, utilizando a desigualdade de Cauchy-Schwarz, concluímos que

$$
\left|\mu^{k_{j}} q\left(x^{k_{j}+1}, x^{k_{j}}\right)\left\langle\zeta^{k_{j}+1}, x-x^{k_{j}+1}\right\rangle\right| \rightarrow 0 \text { quando } j \rightarrow+\infty .
$$

De (5), $f\left(x^{k_{j}+1}, z^{k_{j}+1}\right) \geq f\left(x^{*}, z^{*}\right)$. Portanto, de (7),

$$
f\left(x, z^{*}\right) \geq f\left(x^{*}, z^{*}\right), \forall x \in \Omega .
$$

Mostraremos agora que $x^{*}$ é um solução Pareto para o POM irrestrito (1). Suponha, por contradição, que existe $\bar{x} \in \mathbb{R}^{n}$ tal que $F(\bar{x})<F\left(x^{*}\right)$. Como $z^{*} \in \mathbb{R}_{+}^{m}$, por (P3)',

$$
f\left(\bar{x}, z^{*}\right)<f\left(x^{*}, z^{*}\right) .
$$

Como $\Omega^{k+1} \subseteq \Omega^{k}, \forall k \geq 0$ e $x^{k_{j}} \in \Omega^{k_{j}-1}, \forall j$ com $x^{k_{j}} \rightarrow x^{*}, j \rightarrow+\infty$, temos que $x^{*} \in \Omega$, i.e., $F\left(x^{*}\right) \leq F\left(x^{k}\right), \forall k \in \mathbb{N}$. $\operatorname{Logo} F(\bar{x}) \leq F\left(x^{k}\right), \forall k \in \mathbb{N}$, i.e, $\bar{x} \in \Omega$, o que contradiz (8) e (9).

\section{Conclusões}

Dada a importância das soluções eficientes de problemas multiobjetivo associados a aplicações reais, investigamos e mostramos que os algoritmos proximais de Gregório e Oliveira [1] e Rocha et al. [6] convergem para soluções eficientes.

\section{Referências}

[1] R. Gregório and P. R. Oliveira. A Logarithmic-quadratic proximal point scalarization method for multiobjective programming, J. Global Optim., 49:281-291, 2011.

[2] J. Jahn. Theory of vector maximization: Various concepts of efficient solutions. In: Gal, T., Stewart, T.J., Hanne, T. (eds.): Multicriteria Decision Making. Advances in MCDM Models, Algorithms, Theory, and Applications, volume 21, Kluwer, Boston, pages 37-68, 1999.

[3] K.M. Miettinen. Nonlinear multiobjective optimization. Kluwer, Boston, 1999.

[4] B.S. Mordukhovich. Variational analysis and generalized differentiation I, Grundlehren der Mathematischen Wissenschaften, volume 330, Springer-Verlag, Berlin, 2006.

[5] F.G. Moreno, P.R. Oliveira and A. Soubeyran. A proximal Algorithm with Quasi Distance. Application to Habit's Formation, Optimization, 61:1383-1403, 2011

[6] R.A. Rocha, P.R. Oliveira, R.M. Gregório and M. Souza. Logarithmic quasi-distance proximal point scalarization method for multi-objective programming. Appl. Math. Comput., 273:856-867, 2016.

[7] R.T. Rockafellar and R.J-B Wets. Variational Analysis, Springer, Berlin, 1998.

[8] D.J. White. A bibliography on the applications of mathematical programming multiple-objective methods, J. Oper. Res. Soc., 41(8): 669-691, 1990. 